Trauma Berufskrankh 2005 · 7[Suppl 1]:S78-S84 DOI 10.1007/s10039-004-0945-y

Online publiziert: 8. September 2004

(c) Springer Medizin Verlag 2004

\author{
J. Jerosch $\cdot 0$. Vollmert \\ Klinik für Orthopädie und Orthopädische Chirurgie, \\ Johanna-Etienne-Krankenhaus, Neuss
}

\title{
Arthrodeseverfahren am oberen Sprunggelenk
}

A Sprunggelenks treten gehäuft nach Verletzungen im Rahmen von Freizeit- und Arbeitsunfällen auf. Durch zunehmenden Anspruch an eine aktive Lebensführung und bleibende Mobilität auch im höheren Lebensalter erscheinen dauerhafte Lösungen zur Versorgung arthrotisch veränderter oberer Sprunggelenke attraktiv. Auch werden Verletzungen des oberen Sprunggelenks aufgrund von gesteigerter Unfallhäufigkeit in Freizeit, Sport und Straßenverkehr immer häufiger beobachtet.

$\mathrm{Zu}$ den typischen Verletzungsformen zählen neben höhergradig offenen Frakturen mit meist sehr langwierigen Verläufen die Gelenkfrakturen des Pilon tibiale sowie des Talus. Trotz hoher Versorgungskapazitäten und adäquater Osteosyntheseverfahren führen solche Verletzungsformen aufgrund bleibender Inkongruenzen nicht selten zur verfrühten posttraumatischen Arthrose.

Aufgrund der sich einstellenden klinischen Symptomatik mit rezidivierenden Synoviotiden mit Gelenkergüssen, die später nicht selten auch in Ruhe auftreten, ist die Lebensqualität betroffener Patienten stark eingeschränkt.

Die Arthrodese des oberen Sprunggelenks als etabliertes Operationsverfahren hat trotz des Einsatzes alternativer Behandlungsmethoden einschließlich der Sprunggelenkarthroplastik insbesondere bei jüngeren und aktiven Patienten ihren hohen Stellenwert für die Therapie primärer und sekundärer Arthrosen beibehalten.

\section{Historischer Überblick}

Der Begriff Arthrodese entstammt dem Griechischen und meint eine feste Gelenkbindung. Historisch kam zunächst die Arthrodese am Kniegelenk bei Beinlähmungen infolge der Poliomyelitis zum Einsatz. Unterschiedliche Operationsverfahren wurden u. a. von Langenbeck [34] inauguriert. Zu erwähnen ist Albert [1], der den Einsatz der Kniegelenkarthrodese auf die Indikation der Kniegelenkkontraktur nach Infekt ausweitete. Obwohl Albert 1879 erstmals eine Versteifungsoperation des oberen Sprunggelenks bei einem jungen Mädchen beschreibt, wird der Begriff der Arthodese erst später durch einen seiner Mitarbeiter erwähnt.

$\mathrm{Zu}$ Beginn des 20. Jahrhunderts etablierten sich viele unterschiedliche Arthrodesetechniken. Neben dem Kniegelenk wurde die Indikation auf Erkrankungen des Sprunggelenks, der Fußwurzel und angeborener Fußdeformitäten erweitert. Um 1910 kam die von Cramer beschriebene Arthrodese des oberen Sprunggelenks mit Spaninterposition zum Einsatz. Ende der 1920er Jahre wurde die Indikation auch auf Arthrosen des oberen Sprunggelenks erweitert [34]. Das Problem der Fixation der Resektionsflächen wurde erst Anfang der 1950er Jahre mit Einführung der Kompressionsarthrodese - zunächst mittels Spanbügel durch Greifensteiner und später mittels Fixateur etxterne durch Charnley - angegangen [8].

Bleibende Probleme und hohe Komplikationsraten dieser Methoden führten zur Weiterentwicklung interner Fixationsverfah- ren mittels Spongiosschrauben durch Allgöwer. Anfang der 1970er Jahre wurden zusätzliche Osteosyntheseverfahren mit Kompressionswinkelplatten durch Mittelmaier und das Prinzip der Zugschraubenosteosynthese durch Zimmermann [34] bekannt. Eine arthroskopisch assistierte Arthrodese des oberen Sprunggelenks findet erstmals 1983 durch Schneider Erwähnung [17].

In der Literatur werden über 50 verschiedene Arthrodesetechniken beschrieben, die sich in interne und externe Osteosyntheseverfahren einteilen lassen $[4,5,10$, 13, 14, 19, 21, 38, 25, 27, 30, 32, 33, 36, 40]. Zu den internen Verfahren gehören sowohl die Verriegelungsarthrodesen mit ventra-

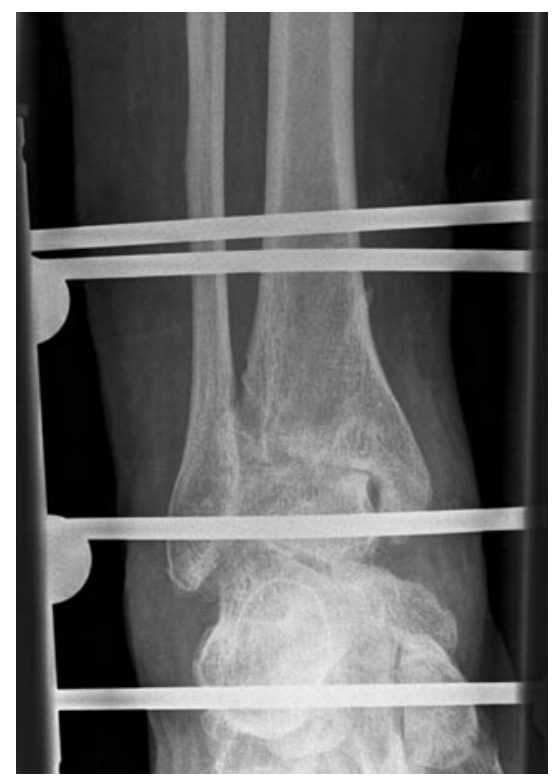

Abb. $1 \Delta$ OSG-Arthrodese mittels Fixateur externe bei ausgeprägter Osteoporose 


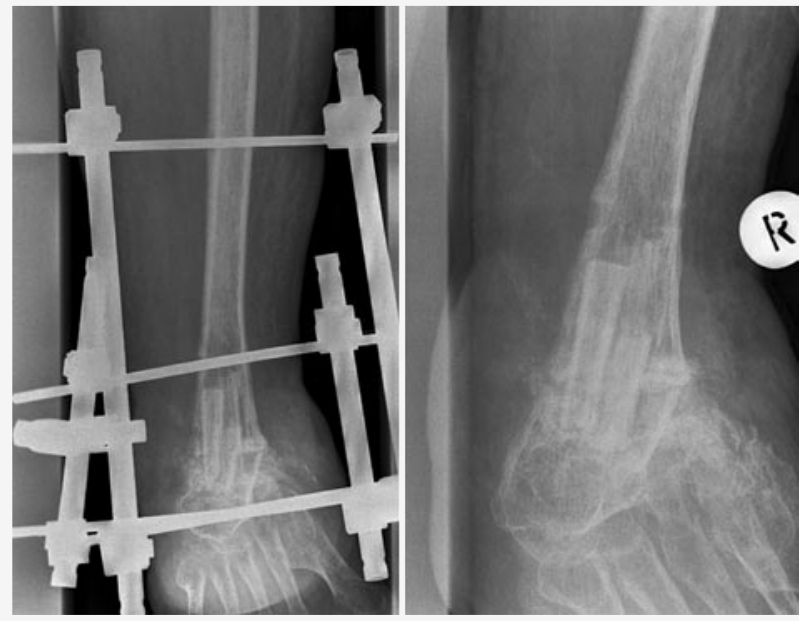

Abb. $2 \Delta$ Ausheilungsergebnis nach Fixateur-externe-Arthrodese bei Zustand nach Infektpseudarthrose mit Fibulaspaninterposition

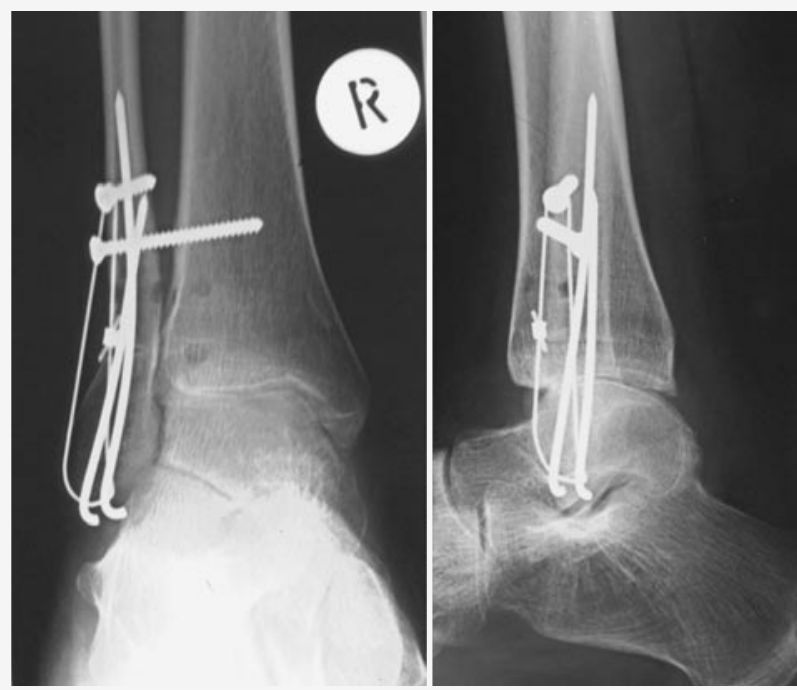

Abb. 4 A Posttraumatische Arthrose des oberen Sprunggelenks nach auswärtiger osteosynthetischer Versorgung

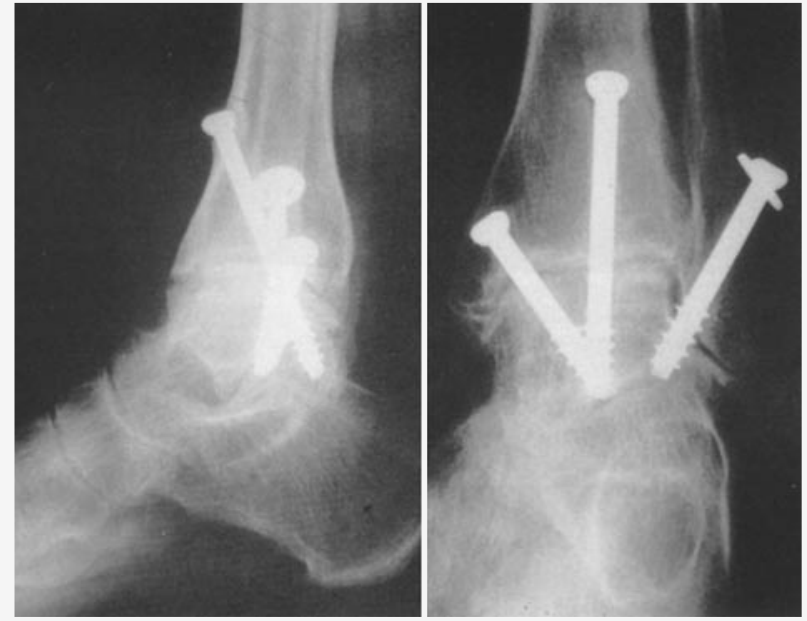

Abb. 3 Zustand nach Arthrodese des oberen Sprunggelenks in 2-Schrauben-Technik mit zusätzlicher ventraler Schraube

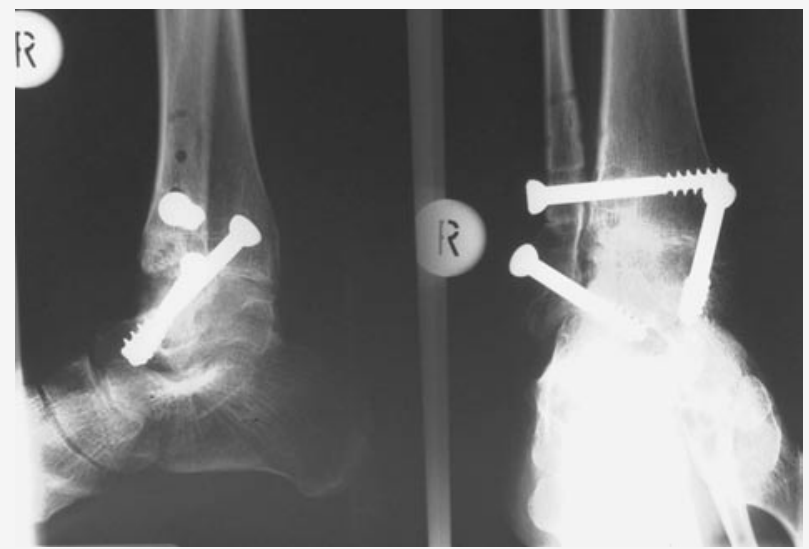

Abb. 5 Kontrolle 6 Monate postoperativ nach Arthrodese des oberen Sprunggelenks in 2-Schrauben-Technik mit zusätzlicher fibulotibialer Schraube lem Tibiaspan oder Fibulatransplantat als auch die Kompressionsarthrodesen durch Verschraubung oder Verwendung von Winkel- oder T-Platten. Trotz Einführung und Verbesserung der Sprunggelenkarthroplastik hat die Arthrodese des oberen Sprunggelenks ihre Bedeutung als Therapie der Wahl bei fortgeschrittenen degenerativen Veränderungen unterschiedlichster Genese bis heute bewahrt. Behandlungsziele sind neben der Schmerzreduktion die Wiederherstellung der Mobilität bei möglichst geringem Funktionsverlust.

\section{Präoperative Diagnostik}

Bevor eine Arthrodese des oberen Sprunggelenks in Frage kommt, sollte neben ei- ner gründlichen Anamneseerhebung eine ausführliche klinische Untersuchung durchgeführt werden. Hierbei werden neben Parametern wie subjektive Beschwerden, schmerzfreie Gehstrecke und bisherige Schuhversorgung auch verschiedene Begleiterkrankungen erfasst. Bei der klinischen Untersuchung sollte dem Gangbild sowie der Gelenkfunktion besondere Beachtung geschenkt werden. Von Bedeutung ist die Erfassung von femorotibialen Achsdeformitäten, die zu Fehlbelastungen des oberen Sprunggelenks führen können. Häufig kann durch eine Korrektur der proximalen Fehlstellung insbesondere bei biologisch jungen und aktiven Patienten mit nur geringgradig ausgeprägter Arthrose eine Arthrodese des oberen Sprunggelenks ver- mieden werden. Zusätzlich sollten konventionelle Röntgenbilder des oberen Sprunggelenks in a.-p.- und seitlichem Strahlengang angefertigt werden. Ferner kommen CT und MRT sowie bei besonderer Indikation die Szintigraphie zum Einsatz [40]. Häufig komplettiert die Arthroskopie die klinische Diagnostik.

\section{Indikationen}

$\mathrm{Zu}$ den wesentliche Indikationen der Arthrodese des oberen Sprunggelenk zählen $[2,3,6,38]$ :

- posttraumatische Arthrosen,

- Arthrosen sonstiger Genese,

- chronische Gelenkinfektionen, 
Trauma Berufskrankh 2005 · 7[Suppl 1]:S78-S84 DOI 10.1007/s10039-004-0945-y

(c) Springer Medizin Verlag 2004

\section{J. Jerosch $\cdot \mathbf{0}$. Vollmert}

\section{Arthrodeseverfahren am oberen Sprunggelenk}

\section{Zusammenfassung}

Die Arthrodese des oberen Sprunggelenks ist weiterhin der Goldstandard in der Behandlung der primären und sekundären tibiotalaren Arthrose. Eine Vielzahl unterschiedlicher Operationstechniken werden in der Literatur beschrieben. Alle Methoden versprechen eine vorhersehbare tibiotalare Fusion. Zu den Komplikationen der Arthrodese des oberen Sprunggelenks zählen Sekundärarthrose in benachbarten Gelenken, die Pseudarthrose und die postoperativen Wundheilungsstörungen sowie die Infektion.

\section{Schlüsselwörter}

Arthrodese - Oberes Sprunggelenk . Tibiotalare Fusion · Sekundärarthrose . Pseudarthrose

\section{Tibiotalar arthrodesis}

\begin{abstract}
Tibiotalar arthrodesis remains the gold standard for the treatment of disabling ankle arthritis. The purpose of this article was to review the different techniques of tibiotalar arthrodesis. Most of these techniques represent predictable methods to obtain fusion of the tibiotalar joint. Complications of upper ankle arthrodesis include arthrosis of adjoining joints, nonunion or malunion, nerve, vessel or tendon injury and infection. With precise surgical technique, appropriate postoperative care, and meticulous patient compliance, stability can be restored to the dysfunctional foot. Arthrodesis of the upper ankle can be extremely rewarding for the patient and surgeon.
\end{abstract}

\section{Keywords}

Arthrodesis - Upper ankle - Fusion of the tibiotalar joint - Arthrosis of adjoining joints . Nonunion
- rheumatische Arthritis,

- Fehlstellungen und Deformitäten infolge paralytischer oder spastischer Lähmung,

- posttraumatische Osteonekrosen (meist in Kombination mit subtalaren Arthrodesen oder als tibiokalkaneare Arthrodese).

Hinsichtlich der Indikationsstellung fließen Parameter wie Gewicht, Alter, Beruf sowie die persönlichen Ansprüche und Erwartungen des Patienten mit in die Entscheidungsfindung ein [32]. Entscheidend sind aber die individuellen Beschwerden des Patienten mit Einschränkung der alltäglichen Leistungsfähigkeit und Lebensqualität in Beruf und Freizeit.

\section{Arthrodeseverfahren}

Abhängig vom Operationszeitpunkt kann zwischen einer Primärarthrodese, einer Früharthrodese sowie einer Spätarthrodese differenziert werden [34].

Die Primärarthrodese kommt in der Regel nur bei ausgedehnten Gelenkfrakturen mit infausten Rekonstruktionsaussichten zum Einsatz und stellt ein technisch hoch anspruchsvolles Verfahren dar.

Eine Früharthrodese wird spätestens 6 Monate nach Trauma durchgeführt. Die Spätarthrodese findet meist bei posttraumatischen oder bei degenerativen Situationen Anwendung. Besondere Berücksichtigung sollten immer auch die Nachbargelenke finden, da ihre physiologische Integrität das funktionelle Ergebnis mit beeinflusst.

Bezüglich der Operationsmethode existieren eine Vielzahl an unterschiedlichen Verfahren, die das Prinzip der Gelenkflächenkompression verfolgen $[27,30]$.

Positive prädiktive Faktoren sind neben einer ausreichenden Kompression der Arthrodeseflächen durch stabile Fixation die Schaffung vitaler spongiöser Oberflächen unter Berücksichtigung der Gelenkflächenkonformität benachbarter Gelenke. [29]. Hinsichtlich der Arthrodeseformen unterscheidet man die Verriegelungsund die Kompressionsarthrodese [34].

Die Verriegelungsarthrodese mit Verriegelungsspan in der Technik nach Campbell oder Wilson [36] beschreibt den Einsatz eines Knochenspans zur Über- brückung des Gelenkspaltes. Auch findet die Verwendung eines Fibulatransplantates in der Technik breite Anwendung. Die reinen Verriegelungstechniken weisen jedoch höhere Komplikationsraten auf und werden zu Gunsten der Kompressionsarthrodesen zurückhaltender eingesetzt. [11]. Indikationen zur Verriegelung bestehen sicherlich bei ausgeprägten knöchernen Defekten oder wenn eine Talusnekrose zu erwarten ist $[3,27]$. Hierzu wird die Verriegelungsarthrodese unter Verschraubung des distalen Fibulaspans mit einer zusätzlichen Kompressionsarthrodese kombiniert. Auch kann eine Verriegelungsspanarthrodese mit anteriorem Tibiaverschiebespan bei Revisionsarthrodesen oder kritischen Durchblutungssituationen zum Einsatz kommen.

Am häufigsten kommen die Kompressionsarthrodesen zur Anwendung. Ziel ist, die Resektionsflächen von Tibia und Talus unter Kompression zubringen, um so eine zügige knöcherne Konsolidierung zu bewirken [2, 38]. Die Methode findet mit interner Fixation durch Schrauben und/oder Platten oder mit externer Fixation mittels Fixateur externe Anwendung $[2,10]$. Voraussetzung ist die sparsame planparallele Entknorpelung oder Resektion der Gelenkflächen [2]. Eine Spongiosaanlagerung wird von einigen Autoren bei bestehenden Gelenkinkongruenzen oder größeren Knochendefekten propagiert. Seltener findet der Fixateur externe aufgrund häufiger Infekte entlang der Steinmann-Nägel und der geringeren Fusionsraten Verbreitung. [2, 12, 27]. Sichere Indikationen für eine externe Fixation finden sich bei Osteoporose im Rahmen neuropathischer Erkrankung oder bei lokalen Infektsituationen (• Abb. 1, 2).

Bezüglich der technischen Durchführung der Kompressionsarthrodese bestehen zahlreiche Modifikationen. Die weiteste Verbreitung findet die Schraubenkompressionsarthrodese, die auch als "golden standard" bezeichnet wird [12, 40]. Die 4-Schrauben-Technik nach Zwipp über einen ventromedianen Zugang beschreibt die Fixation der Gelenkpartner mittels vier 6,5er-Spongiosaschrauben, wobei 2 Schrauben parallel zur distalen Tibia in den medialen und lateralen Taluskörper platziert werden, die 3. Schraube vom dorsalem Innenknöchel in den Taus- 
kopf und zusätzlich eine 4. Schraube transversal vom Außenknöchel in den Talus positioniert wird.

Weiterhin weite Verbreitung findet die von Müller inaugurierte 2-gekreuzteSchrauben-Technik, die zusätzlich mit einer ventralen Antirotationsplatte oder zusätzlicher ventralen Schraube kombiniert werden kann, in ihren zahlreichen Modifikationen ( $\bullet$ Abb. 3, 4, 5). Häufig zu nennende Komplikationen sind versehentliche subtalare Schraubenpositionierungen und die Kreuzung der Schrauben im Arthrodesespalt (• Abb. 6).

Bei ausgeprägter Osteoporose, Osteolysen, Infekten und peripheren arteriellen Durchblutungsstörungen sollten externe Kompressionsarthrodesen diskutiert werden $[38,40]$.

Eine interessante Methode stellt die Arthrodese des OSG mit anterograd oder retrograd eingebrachten Kompressionsmarknägeln dar. Durch ein spezielles Nageldesign ist eine stabile Verriegelung im Talus mit axialer Kompressionsausübung und hoher Primärstabilität möglich [26]. Durch den speziellen Mechanismus kann eine aktive Kompression hergestellt werden, die eine gipsfreie Weiterbehandlung ermöglicht. Die sehr weit distal platzierten Verriegelungsmöglichkeiten bieten eine sichere Verankerung im Talus. Zusätzlich kann eine Spongiosaanlagerung, z. B. in Dübeltechnik, angewendet werden (• Abb. 7).

Die arthroskopisch assistierte Arthrodese (AAA; $[15,17,18,19,28,31])$ des oberen Sprunggelenks mit Minimierung des Zugangstraumas stellt eine interessante Alternative zur konventionellen Arthrodese im Bereich des oberen Sprunggelenks dar. Die weichteilschonende arthroskopische Technik macht diesen Eingriff besonders empfehlenswert für Patienten mit hohem Risiko zu postoperativen Wundrand- und Weichteilnekrosen. Hierzu zählen Patienten mit Gefäß- und Hauterkrankungen, mit Diabetes mellitus und Autoimmunerkrankungen.

Patienten mit starken Formabweichungen der Gelenkpartner im Sinne von Valgus-, Varus- oder Rotationsfehlstellungen und Patienten mit starkem Substanzverlust (aseptische Knochennekrose, große rheumatoide Zysten) sollten nicht in arthroskopischer Technik operiert werden
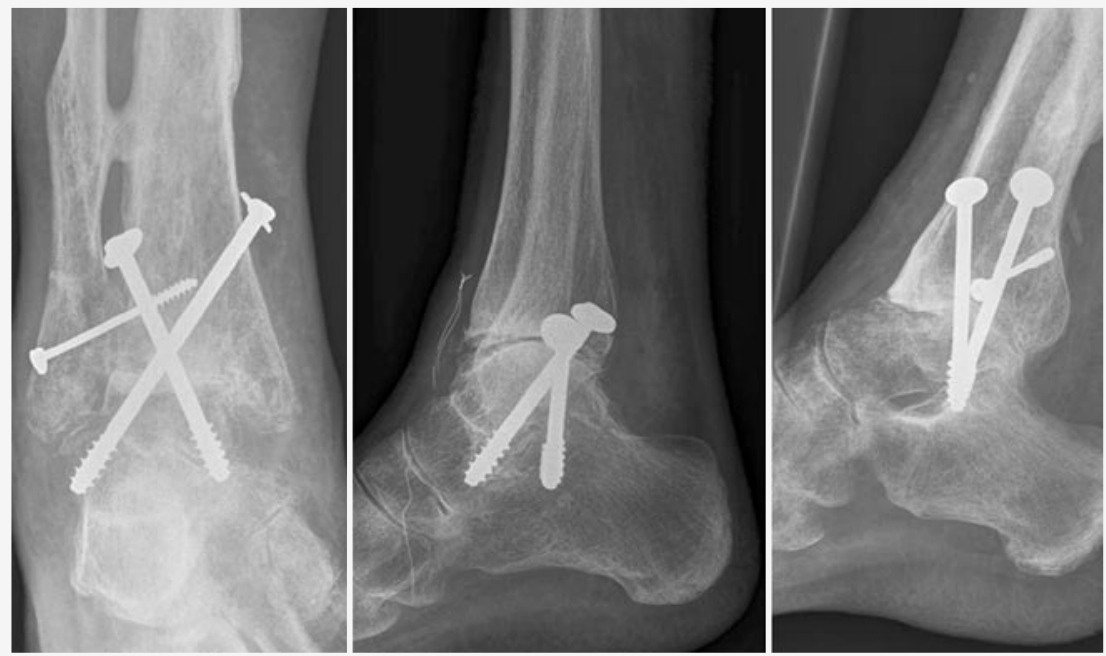

Abb. $6 \Delta$ Typische Schraubenfehllagen bei Arthrodese des oberen Sprunggelenks in 2-Schrauben Technik

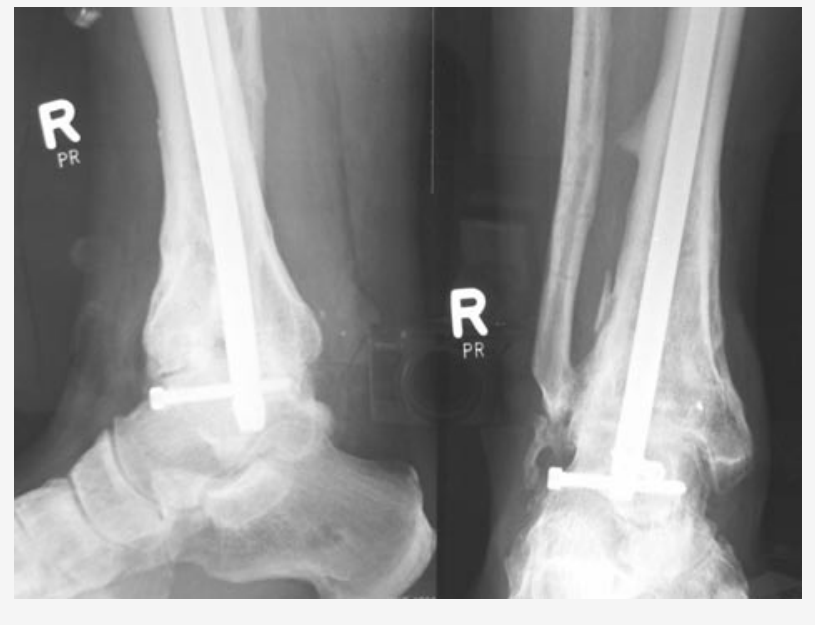

Abb. $7<1$-JahresErgebnis bei Zustand nach posttraumatischer Arthose des oberen Sprunggelenks und Einstellung der Arthrodese mittels anterograd eingebrachtem Kompressionsmarknagel

[19, 28, 31]. Hier muss die Korrektur der knöchernen Deformitäten zusätzlich mit Weichteileingriffen oder Spongiosaplastiken kombiniert werden. In ausgewählten Fällen wurde die Indikation jedoch bereits dahingehend erweitert, dass die AAA mit autologer Spongiosaplastik kombiniert wurde [19]. Auch bei posttraumatischen Arthrosen wird das Verfahren bereits erfolgreich eingesetzt. Aus eigenen $\mathrm{Er}$ fahrungen kann der Autor über gute und sehr gute Ergebnisse bei entsprechender Indikationsstellung berichten ( $\bullet$ Abb. 8, $9,10,11,12$ ).

\section{Komplikationen}

$\mathrm{Zu}$ den mögliche Komplikationen nach Arthrodeseoperationen im Bereich der Sprunggelenke sowie deren Häufigkeiten werden in der Literatur je nach angewand- ter Methode sehr uneinheitliche Angaben gemacht. Zu den am häufigsten auftretenden Komplikationen zählen die Sekundärarthrose in benachbarten Gelenken (o$100 \%)$, die Pseudarthrose (4-35\%) und die postoperativen Wundheilungsstörungen (4-26\%). Seltener werden postoperative Fehlstellungen (2-10\%) und Stressfrakturen (2-8\%) genannt [7, 11, 20, 22, 23, 37, 41].

Konstatierbar ist, dass die Komplikationsraten bei externen Fusionstechniken deutlich höher sind als bei internen Verfahren [41]. $\mathrm{Zu}$ den allgemeinen Risikofaktoren zählen neben dem Diabetes mellitus Durchblutungsstörungen und die Osteoporose.

\section{Nachbehandlung}

Die Nachbehandlung der Arthrodese des oberen Sprunggelenks ist bei Durchsicht 


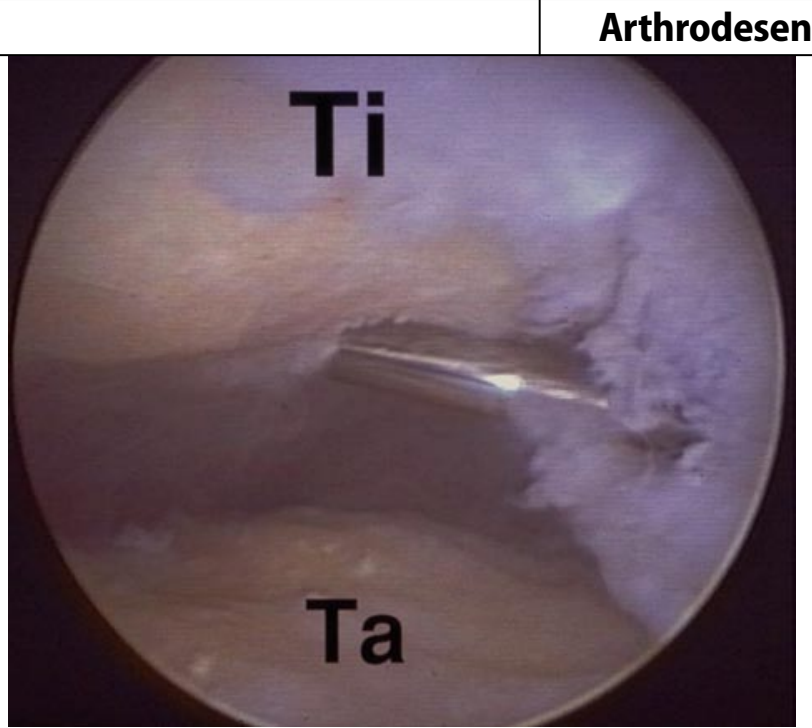

Abb. $8<$ Anbohrung der Tibia bei posttraumatische Arthrose und arthroskopisch assistierter Arthrodese (AAA)

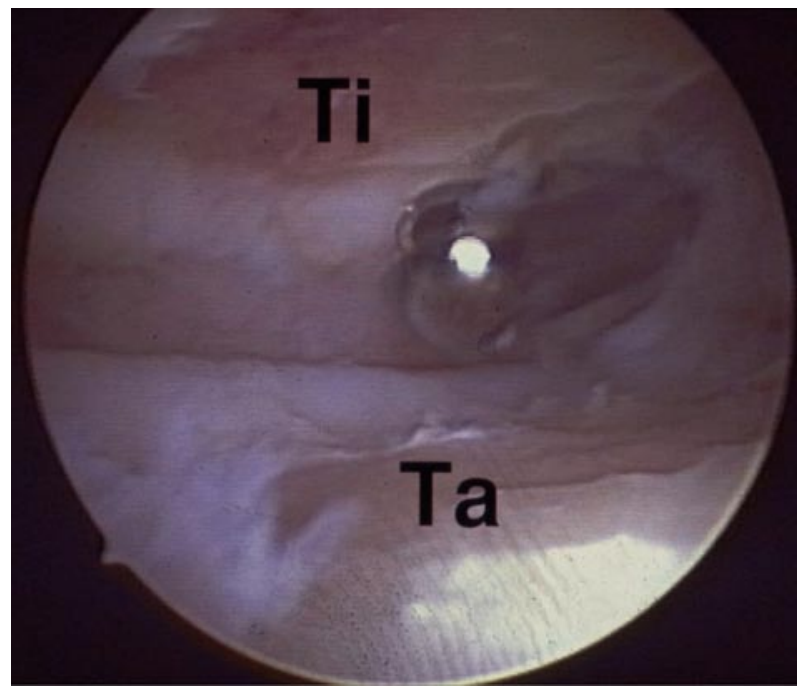

Abb. $9<$ Abrasionschondroplastik zur Vorbereitung der tibialen und talaren Gelenkfläche bei der arthroskopisch assistierten Arthrodese

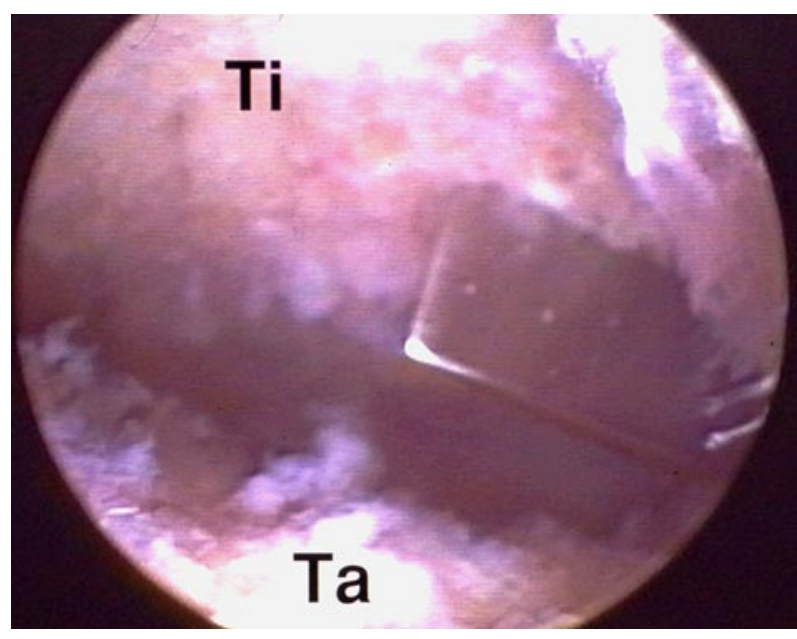

Abb. $10<$ Schaffung kortikospongiöser Oberflächen mittels Meißel bei der arthroskopisch assistierten Arthrodese

der Literatur sehr uneinheitlich. Auch wird die primäre Belastbarkeit der Arthrodese von den einzelnen Autoren unterschiedlich eingeschätzt. So finden sich neben gipsfreien frühfunktionellen Ansät- zen unter Entlastung oder Teilbelastungen $[12,35,40]$ auch restriktive Ansätze mit 6bis 12-wöchiger Immobilisation im Gips ohne jegliche Belastung $[3,32,38,40]$. Subsummierend lässt sich jedoch ein Trend zur eher frühfunktionellen Nachbehandlung bei differenzierten und kooperativen Patienten erkennen. Häufig findet zunächst eine Gipsschienenimmobilisation bis zur gesicherten Wundheilung statt, anschließend kann bei stabiler Arthrodese und kooperativen Patienten eine funktionelle Nachbehandlung mit Solenkontakt erfolgen [38]. Nach der 6. postoperativen Woche wird ein Unterschenkelgehgips für weitere 3-6 Wochen angelegt. Danach erfolgt die Vollbelastung mit entsprechender Schuhversorgung.

Nach Thermann erfolgt bei stabiler Arthrodese die Behandlung gipsfrei und frühfunktionell mit einer Teilbelastung von $15 \mathrm{~kg}$. Nach gesicherter Wundheilung wird für weitere 6 Wochen die Mobilisation im Therapieschuh bei $30 \mathrm{~kg}$ Teilbelastung angestrebt [35]. Bei schlechter Knochenqualität wird die Teilbelastung für 6, bei Verwendung eines Knochenspans für 12 Wochen angeraten.

Allen Behandlungskonzepten gemeinsam ist die subjektive Wertung durch den Operateur. Handelt es sich um eine stabile Arthrodese bei jüngeren und kooperativen Patienten, so ist die Bereitschaft zu einem frühfunktionellem Nachbehandlungskonzept größer.

\section{Diskussion}

Bezüglich der Operationsziele und der Indikation besteht in der Literatur weitgehend Einigkeit. Ziel einer Arthrodeseoperation im Bereich des oberen Sprunggelenks ist neben der Schmerzreduktion die Beseitigung von Instabilitäten sowie die Wiederherstellung einer hinreichenden Mobilität.

Hinsichtlich der anzuwendenden Arthrodesetechnik besteht nach wie vor eine große Meinungsvielfalt, jedoch gilt die Kompressionsschraubenarthrodese als Methode der Wahl [27, 40]. Die breite Streuung der unterschiedlichen Komplikationen einzelner Autoren zeigt, dass eine Standardisierung der operativen Technik noch nicht erfolgt ist. Die meisten Autoren verwenden die 4-Schrauben-Technik oder 2 gekreuzte Schrauben. Bei größeren Defekten oder Osteonekrosen des Talus findet die tibiokalkaneare Arthrodese in Kompressionsschraubentechnik oder mit Fixateur externe vielfach Anwendung. 


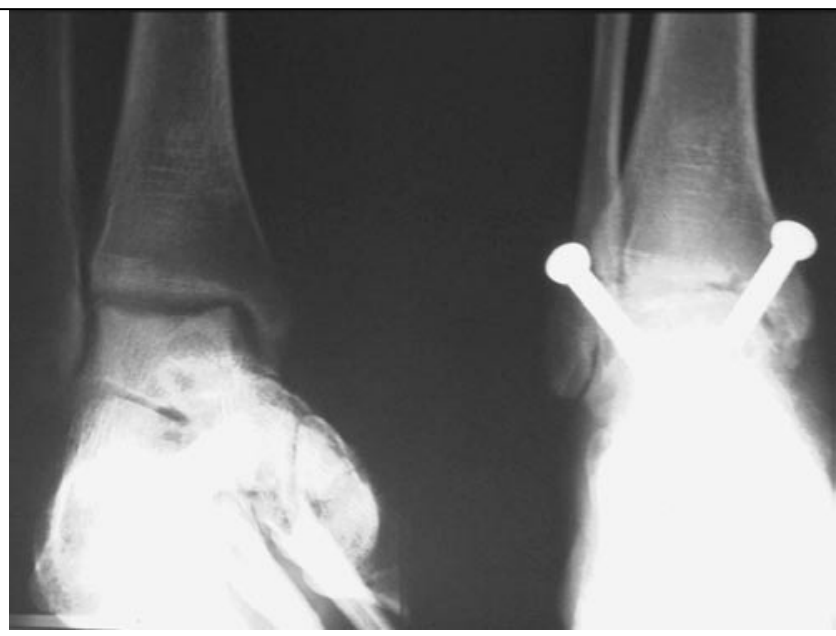

Abb. $11 \Delta$ Prä- und postoperative Situation bei Zustand nach arthroskopisch assistierter Arthrodese

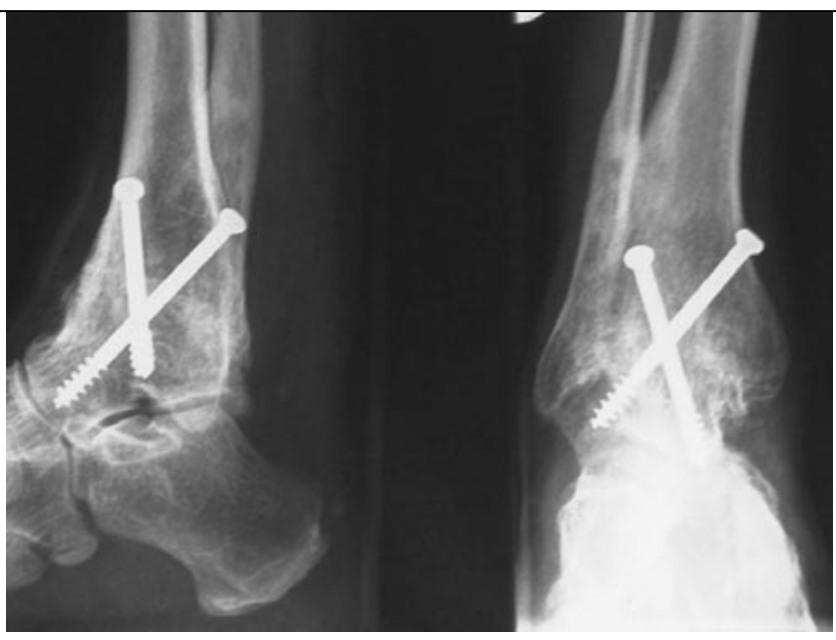

Abb. $12 \Delta$ Ausheilungsergebnis 1 Jahr nach arthroskopisch assistierter Arthrodese
Viele Autoren verwenden zusätzlich eine Spongiosaplastik, insbesondere bei größeren Defekten, Zysten oder Gelenkinkongruenzen.

Die Fußstellung nach Arthrodese hat einen wesentlichen Einfluss auf die postoperativen Langzeitergebnisse [36]. Einheitlich gilt eine Neutralstellung bis maximal $5^{\circ}$ Spitzfuß, ca. $5^{\circ}$ Valgisierung sowie eine Außenrotation des Fußes von 5$10^{\circ}$ im Vergleich zur Gegenseite. Bei experimentellen Untersuchungen fanden sich nach Arthrodese im oberen Sprunggelenk eine deutliche Druckzunahme, insbesondere im Bereich der dorsalen Facette des Subtalargelenks [36]. Der Fuß sollte daher gegen den Unterschenkel um ca. $1 \mathrm{~cm}$ zurückversetzt sein, um durch die Reduktion des anterioren Hebelarms frühzeitigen degenerativen Veränderungen der benachbarten Gelenke - insbesondere des Subtalargelenks - vorzubeugen und ferner ein günstigeres Gangbild zu ermöglichen. Als intraoperative Markierung sollte der vordere Schnitt des Talus mit der Tibiavorderkante abschließen $[38,40]$.

Auch hat sich bei den meisten Autoren das CT gegenüber der konventionellen Röntgendiagnostik zur Frühdiagnostik von Materialfehllagen in der postoperativen Nachsorge durchgesetzt. Hier zeigt sich das Computertomogramm gegenüber konventionellen Röntgenbildern deutlich überlegen, auch wenn zur Beurteilung der Achsverhältnisse oder möglicher Anschlussarthrosen ein konventionelles Röntgenbild ausreicht.

\section{Korrespondierender Autor Prof. Dr. med. Dr. h.c. J. Jerosch}

Klinik für Orthopädie und Orthopädische Chirurgie, Johanna-Etienne-Krankenhaus, Am Hasenberg 46, 41462 Neuss

E-Mail:j.jerosch@jek-neuss.de

Interessenkonflikt: Der korrespondierende Autor versichert, dass keine Verbindungen mit einer Firma, deren Produkt in dem Artikel genannt ist, oder einer Firma, die ein Konkurrenzprodukt vertreibt, bestehen.

\section{Literatur}

1. Albert E (1879) Zur Resektion des Kniegelenkes. Wien Med Press 20: 705

2. Bauer G, Kinzl L (1996) Arthrodesen des oberen Sprunggelenks. Orthopädie 25: 158-165

3. Bauer R, Kerschbaumer F, Poisel S (1995) Orthopädische Operationslehre untere Extremität (2/2). Thieme, Stuttgart

4. Beyaert C, Sirveaux F, Paysant J et al. (2004) The effect of tibio-talar arthrodesis on foot kinematics and ground reaction force progression during walking. Gait Posture 20 (1): 84-91

5. Bier A, Braun H, Kümmel H (1917) Chirurgische Operationslehre. JA Barth, Leipzig

6. Bonnaire F, Cyffka R, Lein T, Hellmund R (2001) Talusfrakturen. Trauma Berufskrankh 3: 192-200

7. Casillas MM, Allen M (2004) Repair of malunions after ankle arthrodesis. Clin Podiatr Med Surg 21 (3): 371-383

8. Charnley J (1951) Compression arthrodesis of the ankle and shoulder. J Bone Joint Surg Br 33B: 180191

9. Chen Y-J, Huang T-J, Shih H-N et al. (1996) Ankle arthrodesis with cross-screw fixation. Acta Orthop Scand 5: 473-478

10. Fitzgibbons TC (1999) Arthroscopic ankle debridement and fusion: indications, techniques and results. Instr Course Lect 48: 243-248

11. Frey C, Halikus NM, Vu-Rose T, Ebramzadeh E (1994) A review of ankle arthrodesis: predisposing factors to non-union. Foot Ankle Int 15:581-584
12. Grass R, Zwipp H (1998) Die Arthrodese des oberen Sprunggelenkes in der Vier-Schrauben-Technik. Operat Orthop Traumatol 10: 134-142

13. Grass R, Rammelt S, Biewener A, Zwipp H (2004) Arthrodesis of the ankle joint. Clin Podiatr Med Surg 21 (2): 161-178

14. Horst F, Nunley JA 2nd (2004) Ankle arthrodesis. J Surg Orthop Adv 13 (2): 81-90

15. Jerosch J, Steinbeck J, Schroder M, Reer R (1996) Arthroscopically assisted arthrodesis of the ankle joint. Arch Orthop Trauma Surg 115 (3-4): 182189

17. Jerosch J, Goertzen M, Reifenrath M (1991) Transarthroskopische Arthrodese des oberen Sprunggelenkes. Indikationen und operative Technik. Arthroskopie 4: 62-65

18. Jerosch J (1999) Arthroskopische Operationen am oberen Sprunggelenk. Indikationen, Technik, Ergebnisse, Komplikationen. Orthopäde 28: 538549

19. Jerosch J (2001) Arthroskopisch assistierte Arthrodese (AAA) des oberen Sprunggelenkes - eine standardisierte OP-Technik. Arthroskopie 14: 205 213

20. Kitaoka HB (1991) Salvage of nonunion following ankle arthrodesis for failed total ankle arthroplasty. Clin Orthop 268: 37-43

21. Kitaoka HB (1999) Arthrodesis of the ankle: technique, complications and salvage treatment Instr. Course Lect 48 255-261

22. Kitaoka HB, Patzer GL (1989) Arthrodesis for the treatment of arthrosis of the ankle and osteonecrosis of the talus. J Bone Joint Surg 3: 370-379

23. Levine SE, Myerson MS, Lucas P, Schon LC (1997) Salvage of pseudarthrosis after tibiotalar arthrodesis. Foot Ankle Int 9: 580-585

24. Mann RA, Rongstad KM (1998) Arthrodesis of the ankle: A critical analysis. Foot Ankle Int 1: 3-969

25. Monroe MT, Beals TC, Manoli A (1999) Clinical outcome of arthrodesis of the ankle using rigid internal fixation with cancellous screws. Foot Ankle Int 4: 227-231

26. Muckley T, Schutz T, Srivastava S et al. (2003) Die Technik der tibiotalaren Arthrodese mit Kompressionsmarknagel. Unfallchirurg 106(9): 732-740

27. Müller EJ, Wick M, Muhr G (1999) Chirurgische Therapie bei Inkongruenzen und Arthrosen am oberen Sprunggelenk. Orthopäde 6: 529-537 


\section{Arthrodesen}

28. O'Brien TS, Hart TS, Shereff MJ et al. (1999) Open versus arthroscopic ankle arthrodesis: a comparative study. Foot Ankle Int 6: 368-374

29. Patterson BM, Inglis AE, Moeckel BH (1997) Anterior sliding graft for tibiotalar arthrodesis. Foot Ankle Int 6: 330-33470

30. Phahler M, Krödel A, Tritschler A, Zenta S (1996) Role of internal and external fixation in ankle fusion. Arch Orthop Trauma Surg 115: 146-148

31. Raikin SM (2003) Arthrodesis of the ankle: arthroscopic, mini-open, and open techniques. Foot Ankle Clin 8 (2): 347-359

32. Rehart S, Peters A, Kerschbaumer F (1999) Arthrodesen am unteren Sprunggelenk beim Erwachsenen. Orthopäde 9: 770-777

33. Rowan R, Davey KJ (1999) Ankle arthrodesis using an anterior AO T-plate. Bone Joint Surg Br 1: 113116

34. Stuhler T (Hrsg) (1994) Arthrodesen. Thieme, Stuttgart, S 229-236

35. Thermann H, Hüfner T, Roehler A, Tscherne H (1996) Schraubenarthrodese des oberen Sprunggelenkes. Orthopädie 25: 166-176

36. Wayne JS, Lawhorn KW, Davis KE, Prakash K (1997) The effect of tibiotalar fixation on foot biomechanics. Foot Ankle Int 12: 792-797

37. Wülker N, Flamme CH, Müller A, Wirth CJ (1997) 10Jahres Verläufe nach Arthrodesen der Rückfußgelenke und des oberen Sprunggelenks. Z Orthop Ihre Grenzgeb 135: 509-515

38. Wülker N, Stepheus M, Cracchiolo A (1998) Operationsatlas Fuß und Sprunggelenk. Enke, Stuttgart, S 245-262 u. 333-344

39. Zgonis T, Jolly GP, Blume P (2004) External fixation use in arthrodesis of the foot and ankle Clin Podiatr Med Surg 21 (1): 1-15

40. Zwipp H (1994) Chirurgie des Fußes. Springer, Wien New York

41. Zwipp H, Grass R, Rammelt S, Dahlen C (1999) Arthrodese - Pseudarthrosen am Sprunggelenk. Chirurg 70: 1216-1224 